\title{
Performing Micro X-ray Fluorescence Analysis in SEM with an Integrated X- ray Generator Based Upon Polycapillary Optic Technology
}

\author{
Ning Gao*, Tom Bievenue*, Ian Radley*, Brian Cross**, and Jim Nicolino*** \\ * X-Ray Optical Systems, Inc., 30 Corporate Circle, Albany, NY 12203 \\ ** CrossRoads Scientific, 414 Av. Portola, P.O. Box 1823, El Granada, CA 94018-1823 \\ *** IXRF Systems, Inc., 15715 Brookford Drive, Houston, TX 77059
}

A compact, low-powered x-ray generator coupled with a polycapillary $\mathrm{x}$-ray optic was integrated into a JEOL scanning electron microscope to provide the system with the capability of micro $\mathrm{x}$ ray fluorescence (XRF) analysis. The excitation $x$-ray beam generated by the combination of the $\mathrm{X}$-ray source and the optic, referred to as X-beam, generates a $25 \mu \mathrm{m}$ focal spot on the sample and a beam intensity of $1 \times 10^{7}$ photon per second at Mo K $\alpha(17.4 \mathrm{keV})$ when the $\mathrm{X}$-ray generator was operated at only ten watts. The flux density of the excitation $\mathrm{x}$ rays on the sample reached to $1 \times 10^{10}$ photon $/ \mathrm{sec} / \mathrm{mm}^{2}$, which was more than three orders of magnitude higher than that obtained without using X-ray optic. The add-on X-beam extended the capability of SEM and improved the detection sensitivity of $\mathrm{x}$-ray analysis for a variety of applications.

Electron-probe x-ray fluorescence (EPXRF) analysis has been widely used in SEM system. The major advantage offered by the electron excitation is the small probe size, typically less than one micron, and its high excitation efficiency, especially in low-energy range. The EPXRF analysis, however, has certain limitations. First, the electron source causes a high bremsstrahlung background, which limit the sensitivity of EPXRF analysis. Secondly, the EPXRF is a surfacesensitive technology and cannot be used to measure underlayer materials. In contrast, XRF analysis offers much higher detection sensitivity, especially for relatively high-energy x rays. Underlayer analysis is possible due to the much better penetrating ability of x rays in comparison with the electron probe. Furthermore, XRF analysis will allow the sample to be measured "as is", while certain level of sample preparation is required for EPXRF. The add-on XRF analysis capability will be particularly useful in low-vacuum SEM (LV-SEM) and environmental SEM (ESEM) systems, where the electron spreading due to the increased chamber pressure dramatically reduces the sensitivity and spatial resolution for x-ray analysis [1].

Although the X-beam cannot provide the spatial resolution the electron beam provides in X-ray analysis, we found the $25 \mu \mathrm{m}$ excitation beam size were appropriate for a variety of applications. The small x-ray beam and the high bema intensity were achieved by using a polycapillary focusing $x$-ray optic $[2,3]$. As illustrated in figure 1 , such an optic collects $x$ rays over a large solid angle from a microfocus $\mathrm{x}$-ray source and redirect them by multiple external total reflections to form a focused beam. In comparison with using a conventional pinhole collimator, the x-ray flux density at the sample plane was dramatically increased. The polycapillary optic used in our work consisted of hundreds of thousands of glass channels with diameter of 10 microns. Figure 2 shows typical microscope pictures of the cross section of such optics. The optic has high transmission efficiency over a wide range of energy (100eV to $25 \mathrm{keV})$.

The polycapillary optic was assembled in a stainless steel tube and was pre-aligned with the Xray source to form the X-beam package. The X-beam was then mounted on a flange that was 
designed to fit into one port of the sample chamber of a JEOL-5610 SEM system. Manual X-Y translation stages were integrated into the mounting flange so that the X-beam could be moved around to overlap with the center of the electron probe.

A fundamental-parameter quantitative analysis software was developed and integrated to the SEM system to allow quantitative XRF analysis. The software took into account the transmission function of the polycapillary optic in the excitation beam spectrum.

Different samples were analyzed using both EPXRF and XRF on the JEOL5610 system and the detection sensitivity were compared to each other. The results

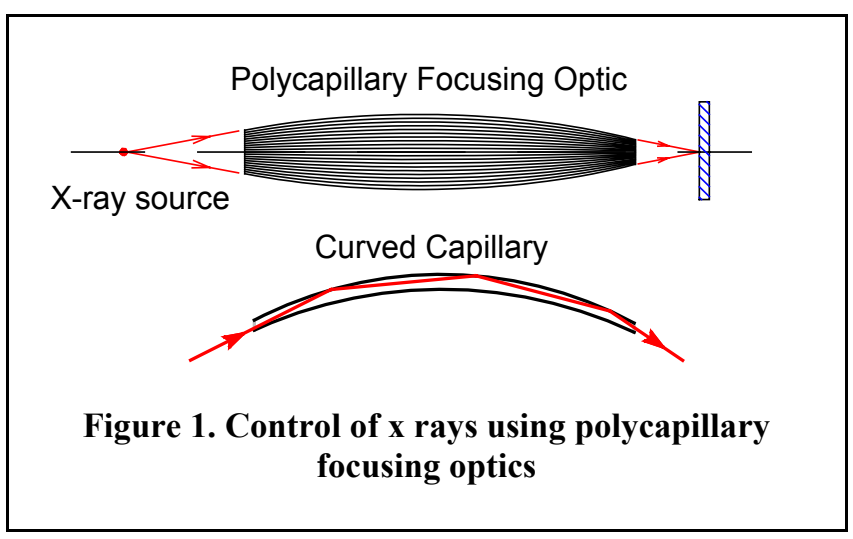
will be reviewed and discussed.

[1] G. D. Danilato, Foundations of environmental scanning electron microscopy, Ad Electronics Electron Physics 71, pp. 109-250 (1988)

[2] Y. Yan and X. Ding, Nucl. Instrum. and Meth., B82, 121-124 (1993).

[3] N. Gao et al, Appl. Phys. Lett., 69, 1529-1531 (1996)

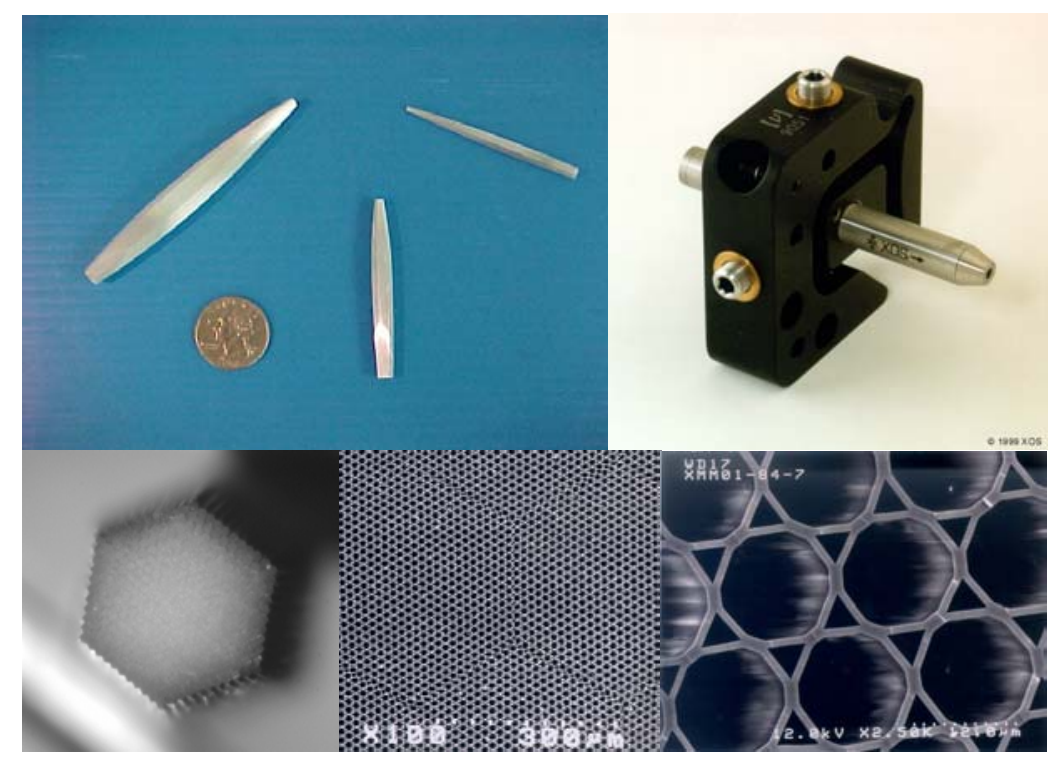

Figure 2. Photographs (above) showing the typical size of polycapillary optics and an alignment jig that holds an optic assembled in a stainless steel enclosure, and optical and SEM images (below) showing the crosssection of a polycapillary optic 